\title{
Tandem effects of lipid head and tail chemistry on clay wettability
}

B. L. KeSSENICH ${ }^{1}$, N. POKHREL ${ }^{1}$, J. KIBUE ${ }^{2}$, M. FluRY ${ }^{3}$, L. MAIBAUM ${ }^{1}$, J. J. De YoREO ${ }^{2,4}$

${ }^{1}$ University of Washington, Department of Chemistry

${ }^{2}$ University of Washington, Department of Materials Science and Engineering

${ }^{3}$ Washington State University, Department of Crop and Soils Science

${ }^{4}$ Pacific Northwest National Laboratory, Physical Sciences Division

Amphiphilic molecules have been implicated in seasonal shifts in soil water repellency, though the underlying mechanisms are unknown. The complexity of natural soils further complicates determining the causes of water repellency. Therefore, we developed simple systems of montmorillonite clay and phospholipids to determine the influence of lipid chemistry on wettability. We combined techniques ranging from molecular (simulations) to nanoscopic (atomic force microscopy) to microscopic (fluorescence microscopy) to macroscopic (contact angle measurements) to explore interactions at all length scales. The wettability was assessed from initial contact angle and time-dependent changes in droplet shape. Our results show that static contact angles do not reflect the key change in the clay films associated with their wettability. Rather, the time dependent decrease in contact angle shows changes in the characteristic time constant for imbibition of water into the film and the degree to which the fluid index of the water deviates from that of a Newtonian fluid. We find that lipid head-group chemistry determines how the lipid binds to the mineral surface and is in turn related to changes in wettability. Whether a lipid is liquid or solid is also related to wettability, as the lipid state changes its distribution through the sample film. Overall, among the lipids tested, those which are solid, bind to the mineral surface, and are distributed through the sample have the largest impact on how water interacts with the model system. 